\title{
Doxycycline Therapy for Abdominal Aortic Aneurysm: Inhibitory Effect on Matrix Metalloproteinases
}

Smit Paghdar ${ }^{1,2}$, Taheseen M. Khan ${ }^{1}$, Nishant P. Patel ${ }^{3,4}$, Savitri Chandrasekaran ${ }^{4}$, Joaquim Francisco Maria De Sousa $4,5,6$, Nicholas Tsouklidis 7, 1, 8

1. Medicine, California Institute of Behavioral Neurosciences \& Psychology, Fairfield, USA 2. Internal Medicine, Surat Municipal Institute of Medical Education and Research (SMIMER), Surat, IND 3. Internal Medicine, Government Medical College, Surat, Surat, IND 4. Internal Medicine, California Institute of Behavioral Neurosciences \& Psychology, Fairfield, USA 5. Surgery, S.S. Institute of Medical Sciences and Research Centre, Davangere, IND 6. Emergency Medicine, Healthway Hospital, Panaji, IND 7. Health Care Administration, University of Cincinnati Health, Cincinnati, USA 8. Medicine, Atlantic University School of Medicine, Gros Islet, LCA

Corresponding author: Smit Paghdar, paghdarsmit@gmail.com

\begin{abstract}
Abdominal aortic aneurysm (AAA) is a life-threatening condition associated with smoking, aging, atherosclerosis, and destruction of the connective tissue in the abdominal aortic wall. Disturbances in the synthesis and degradation of matrix metalloproteinase (MMP) have been known to contribute to the development of AAAs. The only available treatment of AAA is surgical repair. Doxycycline, a tetracycline analog, is thought to have an inhibitory effect on MMPs. Knowing the effect of doxycycline, there may be some favorable effects of the drug to reduce the growth of small AAAs and avoid the need for invasive treatment. This article aims to determine the relationship between doxycycline and the MMPs to prevent the growth of small AAAs. We conducted our review using online resources such as PubMed, Google Scholar, The Journal of Vascular Surgery, and ResearchGate. The result of our study supports the effect of doxycycline in preventing the growth of small AAAs. We conclude that therapeutic treatment with doxycycline in patients with small AAAs can prevent the growth of aneurysms, life-threatening aneurysm rupture, and reduce the need for expensive, invasive treatment.
\end{abstract}

Received 03/06/2021 Review began 04/22/2021 Review ended 05/05/2021 Published 05/11/2021

๑) Copyright 2021

Paghdar et al. This is an open access article distributed under the terms of the Creative Commons Attribution License CC-BY 4.0., which permits unrestricted use, distribution, and reproduction in any medium, provided the original author and source are credited.
Categories: Cardiac/Thoracic/Vascular Surgery, Cardiology, Internal Medicine Keywords: doxycycline, abdominal, aortic aneurysm, matrix metalloproteinase

\section{Introduction And Background}

Abdominal aortic aneurysm (AAA) is a common and serious medical condition in the elderly, especially among males [1]. Aneurysms are usually asymptomatic until they rupture. Rupture is often life-threatening, with mortality ranging from $85 \%$ to $90 \%$ [2]. AAA is characterized by localized chronic inflammation of the aortic wall [3-6]. They often grow slowly and are characterized by localized dilatation of the abdominal aorta in which the aortic diameter is $\geqslant 3.0 \mathrm{~cm}$ (normal diameter $<3 \mathrm{~cm}$ ). The pathogenesis of AAA includes inflammation and immune response, smooth muscle cell apoptosis, extracellular matrix degradation, biomechanical wall sheer stress, and molecular genetics. Figure 1 shows the pathogenesis of AAA through proteolytic degradation with matrix metalloproteinases (MMPs). 


\section{Cureus}
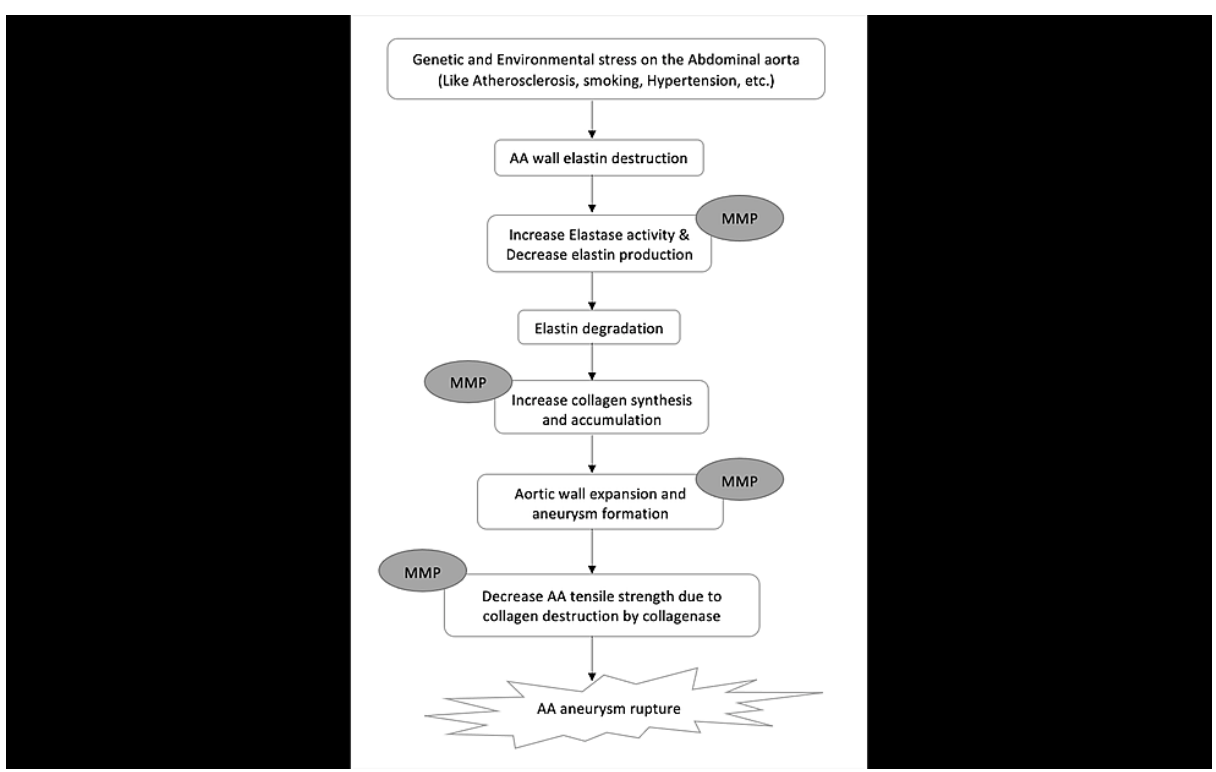

\section{FIGURE 1: Representation of the pathogenesis of AAA through proteolytic degradation with MMPs.}

AAA: abdominal aortic aneurysm; AA: abdomial aorta; MMP: matrix metalloproteinase

The diagnosis of AAA is mostly incidental when a physician scans or examines the abdomen for another reason. Surgical repair is currently the only available treatment option for AAA. A more proactive and noninvasive strategy would be to identify AAAs by screening starting at age 65 , and then to intervene therapeutically to reduce AAA expansion with preventive measures [7]. Small (diameter of $<5.5 \mathrm{~cm}$ ), asymptomatic AAAs are typically managed conservatively by regular monitoring. Current guidelines recommend surgical repair when the aneurysm diameter expands by more than $1 \mathrm{~cm}$ per year or a large diameter of more than $5.5 \mathrm{~cm}$. Nevertheless, surgical treatment may remain the preferred choice of management for patients at a high risk of mortality.

Although elective surgical repair is the only practical approach to prevent deaths from ruptured AAAs, there are no alternative therapeutic strategies for this disease [8]. Because aneurysms are the result of destructive remodeling of the elastic media and outer aortic wall, recent investigations have emphasized disease mechanisms involving chronic aortic wall inflammation and extracellular matrix protein degradation $[9,10]$.

Elastin is a major structural component of the aorta and one of the most substantial proteins of the extracellular matrix [11]. Specific proteinases dissolve elastic fibers, and MMPs are thought to contribute to aneurysm development, including MMP-2, MMP-9, and MMP-12 [12-15]. MMP-9 is the most abundant proteinase secreted by human AAA tissue explants in vitro. It is actively expressed by macrophages located at the site of tissue damage in situ [15]. MMP-9 correlates with increasing aneurysm diameter [16], and its level is elevated in the circulating plasma of patients with AAAs [17]. MMP-12 is also considered important in aneurysm development because it is selectively produced by macrophages within AAA tissue. Unlike other MMPs expressed in the degenerating aortic wall, it is specifically localized to elastin fiber fragments by immunohistochemistry [15]. These observations have speculated that either MMP-9 or MMP-12 might be necessary for aortic wall degeneration and the development of an aneurysm, thereby providing targets for non-invasive pharmacologic therapy $[8,10]$.

The concept of pharmacologic treatment is to prevent the growth of AAA or even reducing aneurysm size and avoiding rupture of an aneurysm. Some studies have indicated that tetracycline derivatives have the ability to stop the progressive degradation of elastin by inhibiting MMPs in the aortic wall, and they do so at clinically approved dose schedules. These study results support the view that MMPs are the targets for pharmacological treatment, and especially tetracyclines might be useful in preventing the growth of AAAs. Hence, this article explores the effectiveness of doxycycline in preventing the growth of small AAAs through several clinical trials and review articles.

\section{Review}

\section{Methodology}

PubMed, Google Scholar, Journal of Vascular Surgery, and ResearchGate online databases were used for the purposes of collecting corresponding data. A total of 48 scientific studies resulted from our search of 
keywords. Keywords included “Doxycycline, abdominal, aortic aneurysm, matrix metalloproteinase.” Of the 48 studies, five were included in our final review article with specific inclusion criteria such as study period between 2000 and 2020, clinical trials, and systemic reviews. All of the included studies met the quality specification and were peer-reviewed.

\section{Results}

A total of five studies were selected to evaluate the effect of doxycycline on the growth of AAAs. One study differed from the rest of the studies and reported no effect of doxycycline on the growth of AAAs, while other studies reported a positive effect of doxycycline to prevent the growth of small AAAs. Doxycycline can inhibit selective MMP inhibition, which arrests the growth of small AAAs. Table 1 lists the studies used in this review article, which includes conclusions drawn from the respective studies.

\begin{tabular}{|c|c|c|c|c|}
\hline Study & Location & $\begin{array}{l}\text { Study } \\
\text { period }\end{array}$ & Sample & Conclusion \\
\hline $\begin{array}{l}\text { Meijer et } \\
\text { al. [18] }\end{array}$ & Netherlands & 2013 & 286 (Patients with AAA diameter 3.5 to $5 \mathrm{~cm}$ ) & $\begin{array}{l}\text { Dox therapy did not reduce aneurysm growth } \\
\text { and did not change the need for AAA repair } \\
\text { surgery }\end{array}$ \\
\hline $\begin{array}{l}\text { Abdul- } \\
\text { Hussien } \\
\text { et al. } \\
\text { [19] }\end{array}$ & Netherlands & 2008 & $\begin{array}{l}60 \text { (Patients with scheduled elective, open AAA } \\
\text { repair without chronic inflammatory disease or } \\
\text { inflammatory aortic aneurysm) }\end{array}$ & $\begin{array}{l}\text { Short-term dox therapy improves the balance of } \\
\text { proteolytic enzymes through an effect on } \\
\text { neutrophil content on the aortic wall }\end{array}$ \\
\hline $\begin{array}{l}\text { Mosorin } \\
\text { et al. } \\
\text { [20] }\end{array}$ & Finland & 2001 & 32 (Patients with AAA diameter $<5.5 \mathrm{~cm}$ ) & $\begin{array}{l}\text { Favorable effect of dox in the prevention of } \\
\text { growth of small AAAs }\end{array}$ \\
\hline $\begin{array}{l}\text { Curci et } \\
\text { al. [21] }\end{array}$ & $\begin{array}{l}\text { United } \\
\text { States of } \\
\text { America }\end{array}$ & 2000 & 15 (Patients with scheduled elective AAA repair) & $\begin{array}{l}\text { Dox influences the connective tissue } \\
\text { degradation within human aneurysm tissue and } \\
\text { reduces the growth of small AAAs }\end{array}$ \\
\hline $\begin{array}{l}\text { Liu et al. } \\
\text { [22] }\end{array}$ & $\begin{array}{l}\text { United } \\
\text { States of } \\
\text { America }\end{array}$ & 2003 & Human SMC culture & $\begin{array}{l}\text { Dox inhibits MMP- } 2 \text { expression from cultured } \\
\text { human aortic SMCs }\end{array}$ \\
\hline$A A: a b d$ & tal aortic an & irysm; d & studies that met the inclusior & $\begin{array}{l}\text { id exclusion criteria for this revie } \\
\text { smooth muscle cells }\end{array}$ \\
\hline
\end{tabular}

\section{Discussion}

Many studies have been conducted worldwide to understand the anti-inflammatory effect of doxycycline in preventing AAA growth as it is believed that the pathology of AAA is best described as a continuous, localized inflammatory response that is accompanied by excess MMP activity [23]. The purpose of doxycycline therapy is the notion that MMP-9 is actively and critically involved in AAA formation. This notion is supported by an abundance of MMP-9 in growing AAAs, and the observation of the MMP-9 gene arrests AAA formation in animal models of the disease. Animal studies have shown a beneficial effect of doxycycline preventing AAA growth [24]. However, human studies, including clinical trials and cohort studies, provide conflicting evidence.

\section{Doxycycline and Expression of Matrix Metalloproteinase-9}

Studies have found that doxycycline-mediated suppression of AAAs resulted from MMP-9 inhibition [25-29]. A randomized, double-blind, placebo-controlled trial was conducted in 2013 by Meijer et al. [18]. A total of 286 patients with small AAA (aneurysm diameter ranging between 3.5 and $5 \mathrm{~cm}$ ) were randomly assigned and included in the study: 144 in the doxycycline group (daily dose of $100 \mathrm{mg}$ of doxycycline) and 142 in the placebo group. This study aimed to test whether doxycycline inhibits AAA progression in humans. AAA diameter measurements were taken by ultrasonography at the start of the study and at six-, twelve-, and eighteen-month follow-up visits. This study concluded that long-term doxycycline treatment did not prevent aneurysm progression. This unexpected study finding challenges the validity of the existing models of the human AAA. It differs from other studies that support the use of doxycycline to prevent the growth of small AAAs.

Another clinical trial by Abdul-Hussien et al. aimed to study the effect of doxycycline in improving 
proteolytic balance by reducing neutrophil content in the abdominal aortic wall [19]. They randomly assigned 60 patients undergoing elective AAA repair into four groups of low- (50 mg/dL), medium- (100 $\mathrm{mg} / \mathrm{dL}$ ), or high-dose (300 mg/dL) doxycycline versus no medication. The study reported that after two weeks of doxycycline treatment before the aneurysm repair surgery reduces aortic wall MMPs and selectively suppresses neutrophil collagenase and gelatinase (MMP-8 and MMP-9) protein levels. It also increases protein levels of the protease inhibitors tissue inhibitor of MMP-1 and cystatin C. This is confirmed by immunohistochemical analysis of aortic wall aneurysm tissue samples obtained during the surgery, which revealed a $75 \%$ reduction in aneurysm wall neutrophil content than the samples obtained from the placebo group patients. The study concluded that short-term preoperative doxycycline therapy helps reduce the growth of small AAAs, presumably through an effect on aortic wall neutrophil content. Hence, it provides a rationale for doxycycline treatment in patients with small AAAs.

In addition to inhibition of MMPs, eradication of Chlamydia pneumoniae infection with doxycycline has the advantage of preventing the growth of small AAAs. C. pneumoniae infection may be responsible as an initiating or aggravating factor in the process of AAA formation and expansion [30]. A prospective, doubleblind, randomized, placebo-controlled study was conducted by Mosorin et al. [20]. The objective of the study was to investigate the efficacy of doxycycline in reducing the expansion of small AAAs. A total of 32 eligible patients were randomly assigned to receive either doxycycline (150 mg daily) or placebo during a threemonth period and examined during an 18-month period by performing an ultrasound. Outcome measures were grouped into aneurysm expansion rate, the number of patients who had AAA rupture or repair, $C$. pneumoniae antibody titers, and serum concentration of C-reactive protein (CRP). The results of this study suggest that the overall aneurysm expansion rates during the last two six-month periods were significantly lower in the doxycycline group than in the placebo group. Results based on the $C$. pneumoniae antibody titer reveal that elevated IgG titers against $C$. pneumoniae were significantly associated with increased expansion rate in the placebo group. According to the serum concentration of CRP, results suggest that CRP values three months after the doxycycline treatment showed a significant decrease from the baseline values. In contrast, CRP values increased in the placebo group. This study concluded that doxycycline may favorably prevent the growth of the small AAAs and can be useful to avoid the need for surgical treatment. However, the small size of the study cannot justify the use of doxycycline treatment to reduce small AAAs.

Another study was performed by Curci et al. to determine doxycycline suppression of MMPs within human aneurysm tissue and explain the molecular mechanisms underlying this effect [21]. Aneurysm tissues were collected from 15 patients with an AAA. Eight were treated with doxycycline before surgery (100 mg orally twice for seven days) and seven were treated with a placebo. MMP activity was examined using gelatin zymography and immunoblot analysis, while RNA was analyzed using reverse transcription-polymerase chain reaction (RT-PCR). This study included examining the effects of doxycycline on the production of MMP in human THP-1 (THP-1 designates a spontaneously immortalized monocyte-like cell line) mononuclear phagocytes in vitro. The results suggest that doxycycline was associated with a slight reduction (24.4\%) in the activity of MMP-2 utilizing zymography. At the same time, a 2.5-fold decrease in MMP-9 was reported in doxycycline-treated patients using immunoblot analysis. A 5.5-fold (81.9\%) reduction in MMP-9 messenger RNA in doxycycline-treated patients was reported using RT-PCR. They also found that when cultured THP-1 monocytes stimulated with phorbol ester, there was a decrease in MMP-9 protein and mRNA expression after doxycycline exposure in vitro. This study concluded that doxycycline may regulate connective tissue degradation within AAA tissue by controlling the monocyte/macrophage expression of MMP-9 mRNA and suppressing the post-translation activation of proMMP-2. Hence, doxycycline treatment can be useful in preventing the growth of AAAs by MMP inhibition.

\section{Doxycycline and Expression of Matrix Metalloproteinase-2}

A study conducted by Liu et al. established the relation between the inhibitory effect of doxycycline on MMP-2 expression from cultured human aortic smooth muscle cells (SMCs) and human aortic aneurysm tissue explant [22]. Human SMC culture was obtained from infrarenal aorta from transplant donors, and monocytes and lymphocytes were separated for the study. Monocytes were cultured for seven days with other co-culture to get a conditioned medium treated with doxycycline at concentrations ranging from 5 to $24 \mathrm{\mu g} / \mathrm{mL}$ for 24 hours. The results show that doxycycline at this concentration directly inhibited MMP-2 production from cultured human aortic SMCs and explanted AAA tissue. They also noted decreased secretion of MMP-9. These findings correlate with the animal study of Petrinec et al. [25]. They found that doxycycline inhibits development in an elastase-induced rat AAA model associated with decreased MMP-2 and MMP-9 production. The study concluded that doxycycline can be useful in clinical trials designed to inhibit the growth of small AAAs and eventually can be offered as a therapeutic treatment for patients with small AAAs to prevent AAA ruptures.

\section{Limitations}

In this review article, the studies did not have a large enough sample size to establish the effect of doxycycline in preventing AAA growth. The safety and side effects of long-term doxycycline use is yet to be proved. In addition, the mode of action of the drug is still unclear. The studies only focus on small-sized AAAs. We would need more clinical trials in the near future, which exclude all limitations and provide solid evidence that we can use doxycycline to prevent the growth of AAAs. 


\section{Conclusions}

In this review article, our aim was to evaluate the effect of doxycycline on preventing the growth of AAAs. Doxycycline has an anti-inflammatory effect by inhibiting the expression of MMP on the wall of the aortic aneurysm, which is responsible for the growth of AAAs. We found that doxycycline improves the balance between MMP degradation and deposition by selectively inhibiting MMP-9 and MMP-2 expression on the aortic aneurysm wall, which reduces the neutrophil content on the wall, thereby reducing its inflammatory effect and preventing the growth of AAAs. We also find that doxycycline reduces the expression of MMP-9 mRNA, which further supports the anti-inflammatory effect of doxycycline. We did not find the exact mechanism of doxycycline preventing the growth of small AAAs. Hence, we conclude that doxycycline prevents the growth of small AAAs (less than $5.0 \mathrm{~cm}$ in size). Thus, we can introduce doxycycline as a therapeutic treatment of small AAAs to prevent the development of a large aneurysm, rupture of a large aneurysm, and prevent the need for invasive treatment of the large aneurysm (more than $5.0 \mathrm{~cm}$ in size), which will benefit patients from life-threatening large aneurysmal rupture, as well as socioeconomically by avoiding the invasive surgery. Further research on this subject is required to strongly support the exact mechanism of action and long-term side effects of doxycycline in the treatment of AAAs. A study with a large sample size is required to evaluate the significant positive anti-inflammatory effect of doxycycline to reduce the growth of AAAs.

\section{Additional Information \\ Disclosures}

Conflicts of interest: In compliance with the ICMJE uniform disclosure form, all authors declare the following: Payment/services info: All authors have declared that no financial support was received from any organization for the submitted work. Financial relationships: All authors have declared that they have no financial relationships at present or within the previous three years with any organizations that might have an interest in the submitted work. Other relationships: All authors have declared that there are no other relationships or activities that could appear to have influenced the submitted work.

\section{References}

1. Lindholt JS: Abdominal aortic aneurysms. Dan Med Bull. 2010, 57:B4219.

2. Brown LC, Powell JT: Risk factors for aneurysm rupture in patients kept under ultrasound surveillance. UK Small Aneurysm Trial Participants. Ann Surg. 1999, 230:289-96. 10.1097/00000658-199909000-00002

3. Ailawadi G, Eliason JL, Upchurch GR Jr: Current concepts in the pathogenesis of abdominal aortic aneurysm. J Vasc Surg. 2003, 38:584-8. 10.1016/s0741-5214(03)00324-0

4. Ernst CB: Abdominal aortic aneurysm. N Engl J Med. 1993, 328:1167-72. 10.1056/NEJM199304223281607

5. Kadoglou NP, Liapis CD: Matrix metalloproteinases: contribution to pathogenesis, diagnosis, surveillance and treatment of abdominal aortic aneurysms. Curr Med Res Opin. 2004, 20:419-32. 10.1185/030079904125003143

6. Thompson RW: Reflections on the pathogenesis of abdominal aortic aneurysms . Cardiovasc Surg. 2002, 10:389-94. 10.1016/s0967-2109(02)00042-x

7. Scott RA, Thompson SG: Screening, surgical repair, and the management of abdominal aortic aneurisms . J Med Screen. 2005, 12:57-8. 10.1258/0969141053908339

8. Thompson RW: Basic science of abdominal aortic aneurysms: emerging therapeutic strategies for an unresolved clinical problem. Curr Opin Cardiol. 1996, 11:504-18. 10.1097/00001573-199609000-00010

9. Dobrin PB, Mrkvicka R: Failure of elastin or collagen as possible critical connective tissue alterations underlying aneurysmal dilatation. Cardiovasc Surg. 1994, 2:484-8.

10. Shah PK: Inflammation, metalloproteinases, and increased proteolysis: an emerging pathophysiological paradigm in aortic aneurysm. Circulation. 1997, 96:2115-7. 10.1161/01.cir.96.7.2115

11. Parks WC, Pierce RA, Lee KA, Mecham RP: Elastin. Adv Mol Cell Biol. 1993, 6:133-82. 10.1016/S15692558(08)60201-5

12. Freestone T, Turner RJ, Coady A, Higman DJ, Greenhalgh RM, Powell JT: Inflammation and matrix metalloproteinases in the enlarging abdominal aortic aneurysm. Arterioscler Thromb Vasc Biol. 1995, 15:1145-51. 10.1161/01.atv.15.8.1145

13. Davis V, Persidskaia R, Baca-Regen L, et al.: Matrix metalloproteinase-2 production and its binding to the matrix are increased in abdominal aortic aneurysms. Arterioscler Thromb Vasc Biol. 1998, 18:1625-33. 10.1161/01.atv.18.10.1625

14. Thompson RW, Holmes DR, Mertens RA, et al.: Production and localization of 92-kilodalton gelatinase in abdominal aortic aneurysms. An elastolytic metalloproteinase expressed by aneurysm-infiltrating macrophages. J Clin Invest. 1995, 96:318-26. 10.1172/JCI118037

15. Curci JA, Liao S, Huffman MD, Shapiro SD, Thompson RW: Expression and localization of macrophage elastase (matrix metalloproteinase-12) in abdominal aortic aneurysms. J Clin Invest. 1998, 102:1900-10. 10.1172/JCI2182

16. McMillan WD, Tamarina NA, Cipollone M, Johnson DA, Parker MA, Pearce WH: Size matters: the relationship between MMP-9 expression and aortic diameter. Circulation. 1997, 96:2228-32. 10.1161/01.cir.96.7.2228

17. McMillan WD, Pearce WH: Increased plasma levels of metalloproteinase-9 are associated with abdominal aortic aneurysms. J Vasc Surg. 1999, 29:122-7. 10.1016/s0741-5214(99)70363-0

18. Meijer CA, Stijnen T, Wasser MN, Hamming JF, van Bockel JH, Lindeman JH, Pharmaceutical Aneurysm Stabilisation Trial Study Group: Doxycycline for stabilization of abdominal aortic aneurysms: a randomized trial. Ann Intern Med. 2013, 159:815-23. 10.7326/0003-4819-159-12-201312170-00007 
19. Abdul-Hussien H, Hanemaaijer R, Verheijen JH, van Bockel JH, Geelkerken RH, Lindeman JH: Doxycycline therapy for abdominal aneurysm: improved proteolytic balance through reduced neutrophil content. J Vasc Surg. 2009, 49:741-9. 10.1016/j.jvs.2008.09.055

20. Mosorin M, Juvonen J, Biancari F, et al.: Use of doxycycline to decrease the growth rate of abdominal aortic aneurysms: a randomized, double-blind, placebo-controlled pilot study. J Vasc Surg. 2001, 34:606-10. 10.1067/mva.2001.117891

21. Curci JA, Mao D, Bohner DG, et al.: Preoperative treatment with doxycycline reduces aortic wall expression and activation of matrix metalloproteinases in patients with abdominal aortic aneurysms. J Vasc Surg. 2000, 31:325-42. 10.1016/s0741-5214(00)90163-0

22. Liu J, Xiong W, Baca-Regen L, Nagase H, Baxter BT: Mechanism of inhibition of matrix metalloproteinase-2 expression by doxycycline in human aortic smooth muscle cells. J Vasc Surg. 2003, 38:1376-83. 10.1016/s0741-5214(03)01022-x

23. Aziz F, Kuivaniemi H: Role of matrix metalloproteinase inhibitors in preventing abdominal aortic aneurysm. Ann Vasc Surg. 2007, 21:392-401. 10.1016/j.avsg.2006.11.001

24. Dodd BR, Spence RA: Doxycycline inhibition of abdominal aortic aneurysm growth: a systematic review of the literature. Curr Vasc Pharmacol. 2011, 9:471-8. 10.2174/157016111796197288

25. Petrinec D, Liao S, Holmes DR, Reilly JM, Parks WC, Thompson RW: Doxycycline inhibition of aneurysmal degeneration in an elastase-induced rat model of abdominal aortic aneurysm: preservation of aortic elastin associated with suppressed production of 92 kD gelatinase. J Vasc Surg. 1996, 23:336-46. 10.1016/s07415214(96)70279-3

26. Curci JA, Petrinec D, Liao S, Golub LM, Thompson RW: Pharmacologic suppression of experimental abdominal aortic aneurysms: acomparison of doxycycline and four chemically modified tetracyclines. J Vasc Surg. 1998, 28:1082-93. 10.1016/s0741-5214(98)70035-7

27. Manning MW, Cassis LA, Daugherty A: Differential effects of doxycycline, a broad-spectrum matrix metalloproteinase inhibitor, on angiotensin II-induced atherosclerosis and abdominal aortic aneurysms. Arterioscler Thromb Vasc Biol. 2003, 23:483-8. 10.1161/01.ATV.0000058404.92759.32

28. Bartoli MA, Parodi FE, Chu J, et al.: Localized administration of doxycycline suppresses aortic dilatation in an experimental mouse model of abdominal aortic aneurysm. Ann Vasc Surg. 2006, 20:228-36. 10.1007/s10016-006-9017-z

29. Turner GH, Olzinski AR, Bernard RE, et al.: In vivo serial assessment of aortic aneurysm formation in apolipoprotein E-deficient mice via MRI. Circ Cardiovasc Imaging. 2008, 1:220-6. 10.1161/CIRCIMAGING.108.787358

30. Lindholt JS, Juul S, Vammen S, Lind I, Fasting H, Henneberg EW: Immunoglobulin A antibodies against Chlamydia pneumoniae are associated with expansion of abdominal aortic aneurysm. Br J Surg. 1999, 86:634-8. 10.1046/j.1365-2168.1999.01126.x 